This paper is published as part of a Dalton Transactions theme issue:

\title{
Dalton Discussion 11: The Renaissance of Main Group Chemistry
}

\author{
Guest Editor: John Arnold \\ University of California, Berkeley, CA, USA \\ 23 - 25 June 2008
}

Published in issue 33, 2008, of Dalton Transactions

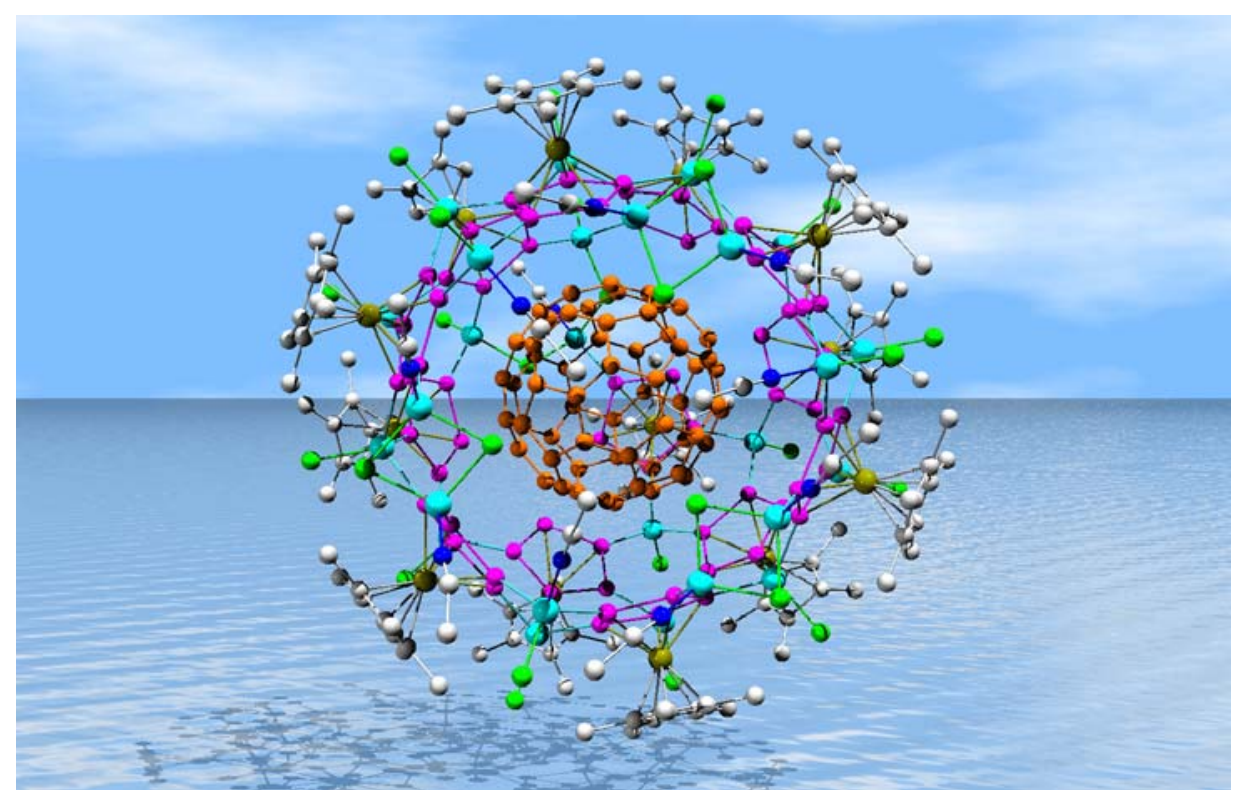

Image reproduced with permission of Manfred Scheer

Papers published in this issue include:

The coordination chemistry of group 15 element ligand complexes-a developing area Manfred Scheer, Dalton Trans., 2008 DOI: 10.1039/b718179p

Formation, structure and bonding of metalloid Al and Ga clusters. A challenge for chemical efforts in nanosciences

Hansgeorg Schnöckel, Dalton Trans., 2008 DOI: 10.1039/b718784j

Polymeric materials based on main group elements: the recent development of ambient temperature and controlled routes to polyphosphazenes Vivienne Blackstone, Alejandro Presa Soto and Ian Manners, Dalton Trans., 2008 DOI: $\underline{10.1039 / \mathrm{b} 719361 \mathrm{k}}$

Recent developments in the chemistry of low valent Group 14 hydrides

Eric Rivard and Philip P. Power, Dalton Trans., 2008 DOI: 10.1039/b801400k

Chemistry and physics of silicon nanowire

Peidong Yang, Dalton Trans., 2008 DOI: 10.1039/b801440j

Visit the Dalton Transactions website for more cutting-edge inorganic and organometallic research www.rsc.org/dalton 


\title{
Effects of ligands and spin-polarization on the preferred conformation of distannynes $\dagger$
}

\author{
Westin Kurlancheek, ${ }^{a}$ Yousung Jung ${ }^{b}$ and Martin Head-Gordon ${ }^{a}$
}

\author{
Received 27th February 2008, Accepted 2nd April 2008 \\ First published as an Advance Article on the web 16th July 2008 \\ DOI: 10.1039/b803417f
}

Recent experimental and theoretical evidence has shown that distannynes, RSnSnR, can adopt either a singly bonded or a multiply bonded structure. Within calculations on small models, such as MeSnSnMe, apparently dramatic differences in conformational preference have been reported. We show that these differences arise due to the treatment of spin-polarization in density functional theory (DFT), and review stability analysis; a diagnostic for the need to include spin-polarization. The low-energy singly bonded structure can only be reached when spin-polarization is allowed. Additional DFT calculations on $\mathrm{PhSnSnPh}$ show that the singly bonded structure is the global minimum, leading to a flat torsional potential. The role of electronic effects is further probed by changing the donor-acceptor properties of R. Implications for the structural preference of experimentally synthesized species are discussed.

\section{Introduction}

Over the past nine years, heavier group 14 homologues of alkynes have been experimentally synthesized and characterized, as fully summarized in recent reviews. ${ }^{1-3}$ The first such compound was a lead analog, ${ }_{4}^{4} \mathrm{Ar} * \mathrm{PbPbAr} *$, where $\mathrm{Ar} *=\mathrm{C}_{6} \mathrm{H}_{3}-2,6-\left(\mathrm{C}_{6} \mathrm{H}_{2}-2,4,6-\right.$ $\left.\operatorname{Pr}_{3}{ }_{3}\right)_{2}$, and $\operatorname{Pr}^{\mathrm{i}}$ is an isopropyl group. Its $\mathrm{PbPb}$ bond length, $3.19 \AA$, is actually slightly longer than the $2.9 \AA$ or so usually associated with $\mathrm{R}_{3} \mathrm{PbPbR}_{3}$ compounds. $\mathrm{Ar}^{*} \mathrm{PbPbAr} *$ is strongly trans-bent, with a $\mathrm{C}-\mathrm{Pb}-\mathrm{Pb}$ bond angle of $94.3^{\circ}$. Subsequently, a corresponding distannyne, $\mathrm{Ar}^{\prime} \mathrm{SnSnAr} \mathrm{Ar}^{\prime},\left(\mathrm{Ar}^{\prime}=\mathrm{C}_{6} \mathrm{H}_{3}-2,6-\left(\mathrm{C}_{6} \mathrm{H}_{2}\right.\right.$ 2,6- $\left.\mathrm{Pr}_{2}^{\mathrm{i}}\right)_{2}$ ) was synthesized and isolated, ${ }^{5}$ with an $\mathrm{SnSn}$ bond length of $2.67 \AA$ (shorter than the typical $2.8 \AA \mathrm{Sn}-\mathrm{Sn}$ single bond), and a substantially larger bending angle of $125^{\circ}$. A corresponding digermyne, $\mathrm{Ar}^{\prime} \mathrm{GeGeAr}^{\prime}$, has been structurally characterized, ${ }^{6}$ which has a GeGe bond length of $2.29 \AA$ ( $c f .2 .44 \AA$ for a single bond) with a trans-bending angle of $129^{\circ}$. An analogous disilyne ${ }^{7}$ shows a very short SiSi bond length of $2.06 \AA$ ( vs $2.34 \AA$ for a single bond) and a yet larger trans-bending angle of $137^{\circ}$. All isolated compounds have been found to be diamagnetic.

The structural trends going up and down group 14 can be qualitatively rationalized on the basis of a changing balance between the contributing resonance structures shown in Scheme 1, which vary between single and triple bonds. For RPbPbR, it appears that resonance form $\mathbf{V}$ dominates, while the doubly bonded forms appear to predominate for $\mathrm{Si}, \mathrm{Ge}$ and possibly for $\mathrm{Sn}$ as well. However, other distannynes have been synthesized which suggest behavior closer to the singly-bonded lead case. The crystal structure of $\mathrm{Me}_{3} \mathrm{Si}-4-\mathrm{Ar}^{\prime} \mathrm{SnSnAr} \mathrm{Ar}^{\prime}-\mathrm{SiMe}_{3}$ has been solved, ${ }^{8}$ and shows a significantly longer $\mathrm{SnSn}$ bond length (3.07 $\AA$ ), and

${ }^{a}$ Department of Chemistry, University of California, and, Chemical Sciences Division, Lawrence Berkeley National Laboratory, Berkeley, CA, 94720

${ }^{b}$ Noyes Laboratory of Chemical Physics, California Institute of Technology, Pasadena, CA, 91125

$\dagger$ Based on the presentation given at Dalton Discussion No. 11, 23-25 June 2008, University of California, Berkeley, USA.

\$ Electronic supplementary information (ESI) available: Additional calculations and optimized coordinates. See DOI: 10.1039/b803417f stronger trans-bending effect (angle of $\left.99^{\circ}\right)$. Spectroscopic $\left({ }^{119} \mathrm{Sn}\right.$ NMR and Mössbauer) studies of Ar*SnSnAr* suggest that it also adopts the longer bond length and smaller trans-bending angle, ${ }^{9}$ although the crystal structure could not be obtained due to poor diffraction. Additionally the solution reactivity of the distannynes (and the corresponding lead species) appears to be lower than the digermynes, based on a comparative study using the $\mathrm{Ar}^{\prime}$ substituent. ${ }^{10}$ This could be consistent with lower contributions from the diradical resonance structure (IV in Scheme 1) for Sn and $\mathrm{Pb}$.

The fact that the structural properties of the tin species are sensitive to substituents suggests that electronic and steric effects can play a crucial role in determining such properties. Electronic structure calculations that predate the experiments support this possibility. ${ }^{11-13}$ Bulky substituents can protect a reactive core, and provide electronic stabilization as well, but may impose steric requirements. They can also probe what happens in the absence of such substituents. For example, calculations (e.g. ref. 14) have shown that unsubstituted HEEH compounds display strikingly different structures to the experimentally isolated compounds, including structures with bridged hydrogens. However, for experimentally isolated molecules, it is only for the tin case that two distinct isomeric forms have been isolated to date.

Recent calculations on the tin system support the possibility that $\mathrm{Ar}^{\prime} \mathrm{SnSnAr}$ may in fact exhibit two local minima corresponding to singly (S) and multiply (M) bonded structures. ${ }^{15}$ The lower energy of the two isomers is the $\mathbf{M}$ form, suggesting it should be favored. This would then suggest that crystal packing forces must account for the $\mathbf{S}$ geometry adopted by $\mathrm{Me}_{3} \mathrm{Si}-4-\mathrm{Ar}^{\prime} \mathrm{SnSnAr} \mathrm{Sr}^{\prime}-4-\mathrm{SiMe}_{3}$ and implied for $\mathrm{Ar}^{*} \mathrm{SnSnAr*}$. Intriguingly, recent calculations on Ar*PbPbAr* have found that two distinct local minima (both an $\mathbf{S}$ and a twisted $\mathbf{M}$ isomer) likewise exist for this compound. ${ }^{16}$ In contradiction to the crystal structure, ${ }^{4}$ the $\mathbf{M}$-type isomer is found to be slightly $\left(\sim 1 \mathrm{kcal} \mathrm{mol}^{-1}\right)$ more stable. ${ }^{16}$ It is clear the potential surfaces of these systems are both rich and delicate, and are fertile ground for further study.

Calculations on model systems for the heavier alkyne congeners, ${ }^{17}$ principally MeEEMe, indicate that the potential 


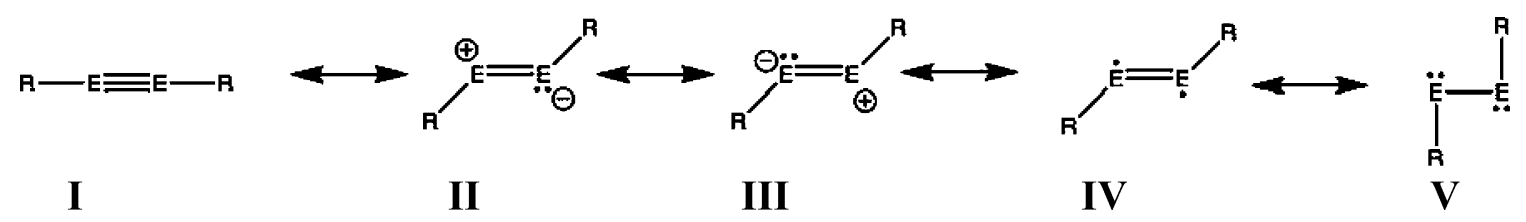

Scheme 1 The possible resonance structures for multiply bonded group 14 compounds.

surface for MeSnSnMe is very flat with respect to bond length and angle distortions away from the local minimum corresponding to an S-type structure towards an M-type geometry. However, the $\mathbf{M}$ structure does not exist as a local minimum, and indeed the global minimum is a gauche-type structure with a dihedral angle of about $90^{\circ}$ (though this will be sterically destabilized with larger substituents). Interestingly, the $\mathbf{S}$-type structure is found to have little diradicaloid character, consistent with low reactivity, while calculations at geometries optimized with the bond-length and trans-bending angle constrained to the experimental $\mathbf{M}$ values, yield significantly higher diradicaloid character, suggesting higher reactivity. This implies that the reactivity differences seen experimentally between $\mathrm{Ar}^{\prime} \mathrm{SnSnAr}{ }^{\prime}$ and $\mathrm{Ar}^{\prime} \mathrm{GeGeAr}^{\prime}$ could be explained by a $\mathbf{S}$-type solution geometry for the tin compound and an $\mathbf{M}$ geometry for germanium. However, a contradictory set of calculations on MeSnSnMe were reported using apparently similar computational methods, ${ }^{15}$ where the $\mathbf{M}$ structure was found to be lower in energy, than the $\mathbf{S}$ structure, which was a saddle point. Further effort to clarify the nature of the potential energy surface for both model and synthesized RSnSnR compounds thus appears desirable.

Accordingly, in this paper we discuss several aspects of electronic structure calculations on the distannynes, and report a variety of new calculations on the model system, $\mathrm{PhSnSnPh}$, which should capture the principal through-bond electronic effects of the more complex and bulky Ar* and $\mathrm{Ar}^{\prime}$ substituents. First, we clarify the origin of differences between the two sets of calculations on the MeSnSnMe model. ${ }^{15,17}$ This requires consideration of the role of spin-polarization in density functional theory calculations, as in fact one set of calculations allowed for spin-polarization and the other did not. Spin-polarization is briefly reviewed in the following section to summarize the key considerations. Second, taking careful account of spin-polarization, we then explore the $\mathrm{PhSnSnPh}$ model in detail to fully characterize its potential surface, and therefore the main through-bond interactions that are operative in the experimentally synthesized distannynes. Finally we consider the effect of additional changes to the electronwithdrawing and electron donating character of the terminal groups, and compare the results obtained with our models against experimentally characterized molecules.

\section{Orbital stability and spin-polarization in density functional theory calculations}

Density functional theory (DFT) in the Kohn-Sham (KS) formalism is the most popular electronic structure method today as it generally yields very good accuracy while requiring little or no more computational effort than mean-field theory. ${ }^{18,19}$ It represents the density of the real system, $\rho\left(\left\{\varphi_{i}\right\}\right)$, using a single determinant wavefunction $\Phi_{0}\left(\left\{\varphi_{i}\right\}\right)$ corresponding to a reference system of non-interacting electrons described by the orbitals $\varphi_{i}$ with same density. This may be problematic when molecules undergo reactions, breaking bonds and forming new ones, or when they may possess some singlet diradicaloid character. In such electronically near-degenerate cases, present-day exchange correlation functionals are not powerful enough to correctly describe multiconfigurational correlation effects in terms of the orbitals of only a single closed shell KS determinant. For example in the simple case of single bond dissociation, the bonding (HOMO) and antibonding (LUMO) orbitals approach degeneracy and so do the two corresponding configurations which have one or the other doubly occupied.

The only way in which standard KS-DFT can reasonably describe bond-breaking is to allow spin-polarization of the orbitals. The resulting unrestricted KS wavefunction has different spatial orbitals for $\alpha$ and $\beta$ spin electrons, which correctly describes most separated products $\left({ }^{3} \mathrm{O}_{2}\right.$ is an interesting exception). For instance a separated single bond has an $\alpha$ electron on one fragment and a $\beta$ electron on the other. This avoids the difficult problem of multiple degenerate electronic configurations that arises with restricted orbitals. It introduces spin-contamination in the reference system, but unlike wavefunction theory, this does not necessarily correspond to real spin-contamination. While the benefits of unrestricted orbitals are obvious at the dissociation limit, it is less obvious for partly broken chemical bonds, such as for diradicaloid molecules. Should they be described by orbitals which are spin-restricted or spin-unrestricted? We advocate always allowing the orbitals to unrestrict (spin-polarize) when this yields a lower energy solution, consistent with the variational principle for Kohn-Sham theory. ${ }^{20}$

The testing of whether or not energy lowering is possible through spin-polarization is called stability analysis. ${ }^{21-23}$ In practice, the KS orbitals $\varphi_{i}$ are obtained by solving the self-consistent field (SCF) equations iteratively until the first variation of the energy with respect to orbital mixings is numerically zero. There is no guarantee, however, that the calculated SCF energy is a true local minimum in the orbital space. The converged energy can be a saddle point, and the KS wave function is then said to be unstable. We will focus on the possible instability of the spin-restricted KS orbitals with respect to release of this constraint (unrestriction) since this is directly relevant for singlet diradicaloid molecules such as models of the distannynes. It is denoted as an $\mathrm{R} \rightarrow \mathrm{U}$ instability and is also called a triplet instability.

Orbital instabilities can be studied by considering response of the energy up to second order, starting from a stationary point, so linear terms vanish:

$$
\Delta E=\frac{1}{2}\left(\begin{array}{ll}
\mathbf{D}^{*} & \mathbf{D}
\end{array}\right)\left(\begin{array}{cc}
\mathbf{A} & \mathbf{B} \\
\mathbf{B} * & \mathbf{A} *
\end{array}\right)\left(\begin{array}{c}
\mathbf{D} \\
\mathbf{D} *
\end{array}\right)
$$


Here $\mathbf{D}$ is a column vector that describes small mixings of each occupied orbital $(i)$ with each virtual orbital $(a)$, so that:

$$
\Phi \cong \Phi_{0}+\sum_{i a} D_{i a} \Phi_{i}^{a}+\ldots
$$

$\Phi^{a}{ }_{i}$ denotes the replacement of orbital $\varphi_{i}$ by $\varphi_{a}$. The precise form of the matrices $\mathbf{A}$ and $\mathbf{B}$ is already available in the literature ${ }^{21-23}$ and is not important for our present purposes.

A necessary and sufficient condition for stability (i.e. for $\Delta E$ in eqn (1) to be positive for all infinitesimal variations, D) is for the square matrix in eqn (1), denoted as $\boldsymbol{\Lambda}$, to be positive definite. For real orbitals (and thus real $\mathbf{A}$ and $\mathbf{B}$ ), the eigenvalues of $\boldsymbol{\Lambda}$ consist of the union of eigenvalues of the two matrices $\mathbf{A}+\mathbf{B}$ and $\mathbf{A}-\mathbf{B}$. Hence if either of those two matrices is not positive definite, the solution found will be unstable. In particular, the SCF orbitals are unstable to unrestricted spin-polarization if $\mathbf{A}+\mathbf{B}$ has a negative eigenvalue (an $\mathrm{R} \rightarrow \mathrm{U}$ instability). A negative eigenvalue of $\mathbf{A}-$ $\mathbf{B}$ indicates the (real) SCF orbitals are unstable to variations that introduce a complex component.

In practice, after an SCF calculation is converged, a test for stability is performed by iteratively finding the smallest (least positive) eigenvalue of the matrices $\mathbf{A}+\mathbf{B}$ and $\mathbf{A}-\mathbf{B}$. Each iteration consists of operations basically similar to a step of the SCF cycle itself (forming a Fock-like matrix), and thus the cost of a stability test typically involves a cost that is not greater than the SCF calculation itself. We think it is advisable to routinely perform such a test. If the result indicates an instability, most computational chemistry programs have the ability to distort the SCF solution along the direction of the instability, and then restart the SCF procedure leading to a lower energy solution. This will then give the lowest DFT energy attainable with the given functional, and for this reason should be preferred. While clearly needed for dissociation, there are also some cases where it is known that significantly different stable structures can be obtained in DFT when spin-polarization is allowed. ${ }^{24}$

\section{Computational methods}

All calculations were performed with a development version of the Q-Chem program. ${ }^{25}$ In this study, all structures were optimized using DFT with the B3LYP functional..$^{26,27}$ Some additional calculations were performed with the B3PW91 functional ${ }^{26}$ to compare with previous work. ${ }^{15}$ As discussed in detail above, all DFT calculations at the restricted level were tested for orbital instabilities, and, if detected were re-optimized using unrestricted DFT. Numerical integration was performed with the standard grid 1 (SG1), ${ }^{28}$ and test calculations were also performed with larger grids to establish that no significant differences occur for delicate energy differences such as the torsional potential energy scans discussed later.

The $6-31 \mathrm{G}^{*}$ basis set was used for all atoms that are not tin in this investigation, which is of medium size. For the tin atoms the CRENBL effective core potential with the CRENBL* basis was used. ${ }^{29}$ This removes a krypton core from explicit consideration, while leaving the $3 d, 4 s, 4 p$ electrons described by (3s3p4d) basis. Some test calculations were also performed using the Huzinaga [433111/433111/43] (spd) all-electron basis set ${ }^{30}$ for comparison. An all-electron calculation neglects relativistic effects on the core electrons, which can have a noticeable effect on calculated properties of systems containing heavier elements. On the other hand ECPs themselves contain some intrinsic errors.

The diradicaloid character of several structures is determined by using the perfect pairing method. ${ }^{31,32}$ In this method, we look at the occupation number of the lowest unoccupied molecular orbital in order to determine the percentage of diradical character ${ }^{33-35}$ If the species is fully diradical, then the LUMO will have an occupation number of 1 . Therefore the percentage diradical character is simply the LUMO occupation number multiplied by 100 ; this procedure has proven useful in comparative studies of diradical character. ${ }^{36}$ All perfect pairing calculations were performed taking all valence electrons to define the active space (e.g. 43 pairs for $\mathrm{PhSnSnPh}$ ).

Localized orbitals are also determined for several structures. The Kohn-Sham energy is invariant to mixing of the occupied amongst themselves, and such mixings may be determined to extremize a measure of orbital locality. We employ the EdmistonRuedenberg criterion, ${ }^{37}$ which maximizes the Coulomb repulsion of all orbitals with themselves (and thereby minimizes the nonclassical exchange). Efficient algorithms for this problem are now available. ${ }^{38,39}$ We shall use these orbitals to discuss the chemical bonding that is implied by the KS-DFT calculations.

\section{Results and discussion}

\section{MeSnSnMe model system}

As discussed in the Introduction, we want to investigate the quite surprising difference in DFT results between two theoretical studies on MeSnSnMe ${ }^{15,17}$ which in turn has some implications for the behavior of distannynes with more complex substituents. In the work of Jung et al..${ }^{17}$ the singly bonded isomer, $\mathbf{S}$, with an $\mathrm{SnSn}$ bond length of $3.06 \AA$, and CSnSn angle of $100^{\circ}$ is the only minimum that has a planar geometry. No shorter bond-length multiply bonded isomer (M) was located as a stationary point. By contrast, in Takagi and Nagase, ${ }^{15}$ an $\mathbf{S}$-like structure is a first-order saddle point while $\mathbf{M}$ is the planar minimum (with bond length $2.61 \AA$, CSnSn angle $126^{\circ}$ ), where $\mathbf{S}$ is $6 \mathrm{kcal} \mathrm{mol}^{-1}$ less stable than $\mathbf{M}$.

It turns out that in one case, ${ }^{17}$ a stable unrestricted DFT solution was used, while in the other case,${ }^{15}$ an unstable restricted DFT solution was used. We find that the structure $\mathbf{M}$ used by Takagi and Nagase ${ }^{15}$ shows a triplet $(\mathrm{R} \rightarrow \mathrm{U})$ instability using the same method used in their study. However, the two studies also used different density functionals, and different basis sets (one is all-electron and the other also uses an effective core potential (ECP) basis). The effect of each of these differences needs to be established. Therefore, we performed the systematic calculations summarized in Table 1.

The B3PW91 functional used by Takagi and Nagase and B3LYP used in Jung et al. yield almost the same results for the Huzinaga all-electron basis used by Takagi and Nagase. RB3PW91 and RB3LYP yield $d=2.61$ and $2.63 \AA$, respectively, and UB3PW91 and UB3LYP yield $d=2.82$ and $2.85 \AA$, respectively. Therefore, the remaining results are obtained using just B3LYP, as shown in Table 1. Differences between the density functionals are not significant, and can be ruled out as a cause of the difference in results.

The next effect we find is that using an all-electron basis, which does not have relativistic effects for $\mathrm{Sn}$, can tend to shorten the SnSn bond length, relative to an effective core potential, 
Table 1 Summary of B3LYP stability analyses for MeSnSnMe with relevant energetic and geometric parameters. See text for references to basis sets

\begin{tabular}{|c|c|c|c|c|c|c|c|c|c|}
\hline$n$ (core) & Basis & $\mathrm{R} / \mathrm{U}$ & $\left\langle\hat{S}^{2}\right\rangle$ & Stable & $\Delta E / \mathrm{kcal} \mathrm{mol}^{-1}$ & $d / \AA ̊$ & $\angle 1^{\circ}$ & $\phi /^{\circ}$ & Imag. freq. \\
\hline \multirow[t]{2}{*}{36} & \multirow[t]{2}{*}{ CRENBL $^{a}$} & $\mathrm{U}$ & 0.94 & $\checkmark$ & -7.7 & 3.06 & 100 & 180 & 0 \\
\hline & & $\mathrm{R}^{e}$ & 0.00 & $x$ & 0 & $2.65^{e}$ & 125 & 180 & $2^{e f}$ \\
\hline & Def2-SVP ${ }^{b}$ & $\mathrm{R}$ & 0.00 & $x$ & 0 & 2.65 & 124 & 180 & $1^{g}$ \\
\hline \multirow[t]{2}{*}{0} & \multirow[t]{2}{*}{ Huzinaga $^{c}$} & $\mathrm{U}$ & 1.01 & $\checkmark$ & -1.9 & 2.85 & 107 & 180 & 0 \\
\hline & & $\mathrm{R}$ & 0.00 & $x$ & 0 & 2.63 & 126 & 180 & 0 \\
\hline 0 & $\mathrm{TZVPP}^{d}$ & $\mathrm{R}$ & 0.00 & $x$ & & 2.64 & 126 & 180 & 0 \\
\hline
\end{tabular}

${ }^{a} \mathrm{Sn}=[3 \mathrm{~s} 3 \mathrm{p} 4 \mathrm{~d}]$ with 36 core electrons. Augmented by a d polarization function for carbon. ${ }^{b} \mathrm{Sn}=[4 \mathrm{~s} 4 \mathrm{p} 2 \mathrm{~d}]$ with 28 core electrons, and $6-31 \mathrm{G}(\mathrm{d})$ for carbon. ${ }^{c} \mathrm{Sn}=[7 \mathrm{~s} 6 \mathrm{p} 4 \mathrm{~d}]$, all electron. ${ }^{d} \mathrm{Sn}=[6 \mathrm{~s} 5 \mathrm{p} 3 \mathrm{~d} 2 \mathrm{f}]$, all electron. Only the restricted result is reported. ${ }^{e}$ Geometry for the planar $\mathbf{M}$ structure at RB3LYP/CRENBL was obtained by imposing a geometric constraint $d(\mathrm{Sn}-\mathrm{Sn})=2.65 \AA$ and optimizing it, since the fully optimized RB3LYP/CRENBL structure is gauche with $d=2.88 \AA, \angle=68^{\circ}$, and $\phi=92^{\circ} .{ }^{f}(51 i, 52 i){ }^{g}(33 i)$.

which implicitly includes relativistic effects. The RB3LYP tintin distance $(2.6 \AA)$, is independent of the basis set used, allelectron or ECP. However, the UB3LYP distance changes from 2.85 to $3.06 \AA$ on going from the Huzinaga (all-electron) ${ }^{30}$ to CRENBL (36 effective core electrons) basis. ${ }^{29}$ Although $2.85 \AA$ is midway between typical $\mathbf{M}$ and $\mathbf{S}$ values in terms of distance, the calculated bending angle suggests that it is closer to the structure S. This effect, therefore, while noticeable, does not account for the striking difference in results. We note that in Table 1 we also test the effect of making the effective core potential describe fewer core electrons through use of the Def2-SVP ECP and basis. ${ }^{40}$ We find this ECP gives qualitatively the same results, although it shows slightly shorter bond lengths. Finally we also examined the effect of using a larger all-electron basis, by using the TZVPP basis. ${ }^{41}$ This does not alter the results obtained with the Huzinaga basis significantly.

The most important point is that the short bond isomer $\mathbf{M}$, which is located as a minimum in RB3LYP, shows a triplet $(\mathrm{R} \rightarrow$ $\mathrm{U})$ instability in all cases, and upon lifting the spin restriction, optimizes to a long bond structure $\mathbf{S}$. The structure $\mathbf{S}$ is more stable than $\mathbf{M}$ by $2-5 \mathrm{kcal} \mathrm{mol}^{-1}$. This is the principal reason that Takagi and Nagase, who used the (unstable) RDFT solutions obtained $\mathbf{M}$ as more stable than $\mathbf{S}$ by $6 \mathrm{kcal} \mathrm{mol}^{-1}$ (their $\mathbf{S}$ structure was also unstable). It must be emphasized that while this explains the main source of the seemingly dramatic difference reported in refs. 15 and 17, it does not establish which (if either) is actually quantitatively correct for this system. Takagi and Nagase tried to address this issue using high-level wavefunction calculations (CASPT2 (6,6)), and reported that $\mathbf{S}$ was $17 \mathrm{kcal} \mathrm{mol}^{-1}$ less stable than M, which appears to support their RDFT results. However such calculations are more difficult to converge with respect to basis set than DFT, which is one possible source of error, and additionally neglected relativistic effects. For the remainder of the paper we shall always permit the DFT calculations to unrestrict whenever this lowers the energy, unless otherwise specified.

\section{PhSnSnPh model system}

The global minimum of the MeSnSnMe model is a gauche-like structure rather than either trans-bent species. To probe further, we attempt to separate steric from electronic effects due to the substituents. While the bulky $\mathrm{Ar}^{\prime}$ ligands may be needed to crystallize this compound, and may play a key role in steric interactions, it is likely that the groups immediately next to the tin atoms should provide the most important electronic effects. Other bulky ligands used in previous experimental studies (e.g. Ar*, $\mathrm{Ar}^{\prime}-4-\mathrm{SiMe}_{3}$ ) have also contained aromatic carbon rings directly attached to the tin core. Therefore, $\mathrm{PhSnSnPh}$ may be the simplest appropriate model system to capture the role of through-bond electronic effects.

An optimization was then performed on the $\mathrm{PhSnSnPh}$ system in order to find the lowest energy structure. It was discovered that there are several distinct minima corresponding to four different structures. Three of these structures are on the spinrestricted orbital surface while the global minimum is on the spinunrestricted surface. Only the gauche-like structure, structure II in Fig. 1, is stable to spin-polarization, all of the other structures decrease in energy if the orbitals are allowed to unrestrict. If
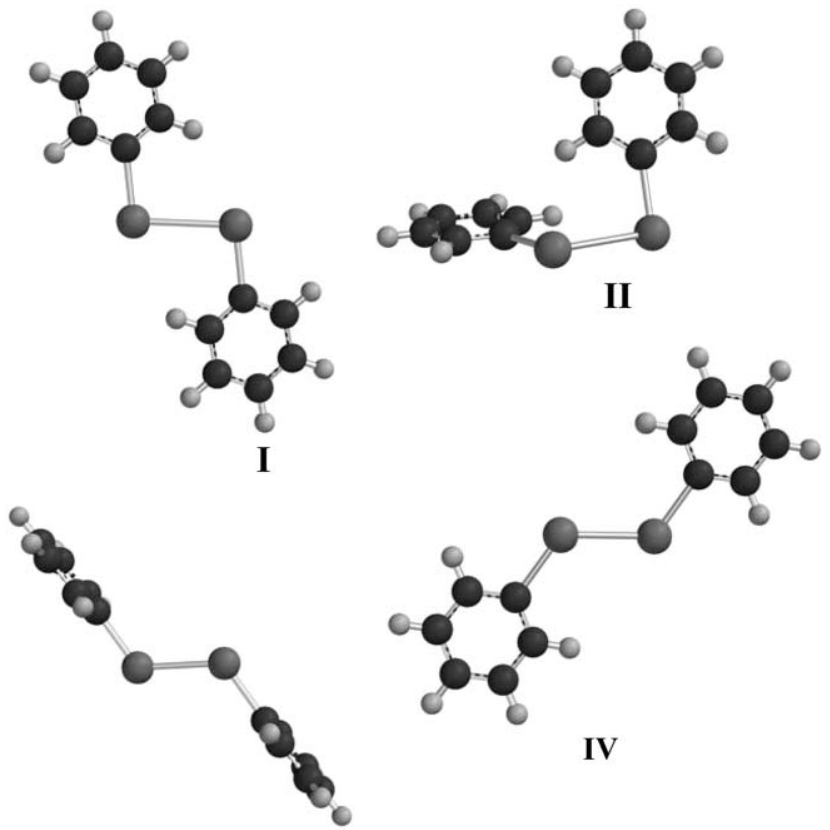

IV

Fig. 1 Optimized $\mathrm{PhSnSnPh}$ structures. Structure I is the planar singly bonded structure. Structure II is the gauche structure. Structure III is the multiply bonded structure, obtained with restricted orbitals (unstable to spin-polarization, but a local minimum on the spin-restricted PES). Structure IV is a multiply bonded structure with the $\mathrm{Ph}$ rings in the CSnSnC plane (unstable to spin-polarization, and also not a local minimum on the spin-restricted PES). 
these calculations had been done without lifting spin symmetry, then the global minimum would have never been uncovered. The global minimum is a planar singly bonded structure shown in Fig. 1 (structure I). This structure has a relatively long $\mathrm{Sn}-\mathrm{Sn}$ bond distance of $3.134 \AA$ and is quite planar around the central Sn-Sn bond with a dihedral angle of $\omega=180.0^{\circ}$. Furthermore, this structure is very trans-bent with a Sn-Sn-C bond angle of $\theta=$ $98.1^{\circ}$. These results agree reasonably well with the results found by Jung et al. for MeSnSnMe, except that the phenyl rings have made a planar structure the global minimum instead of a gauche structure. This is a clear indication of the importance of electronic effects on the preferred conformations of distannynes.

By using a localized orbital procedure, it is possible to explore the character of the bonding and non-bonding orbitals of the central tin atoms. The Edmiston-Ruedenberg localized orbitals (Fig. 2) show one Sn-Sn $\sigma$ bonding orbital and two lone pair orbitals, one on each tin atom. These orbitals, therefore, confirm the singly bonded nature of this molecule. Based on this global minimum structure, it appears that these distannynes should act more like diplumbynes, which adopt a singly bonded structure, rather than digermynes, which adopt a multiply bonded structure. However, given the sensitivity to electronic effects, one should also bear in mind that this character can be quite readily altered by chemical substitution.

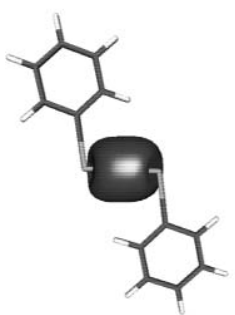

б-bond

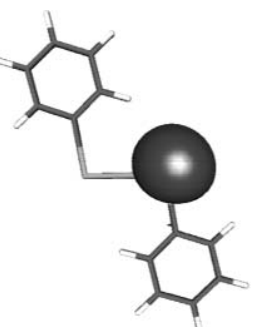

Lone Pair 1

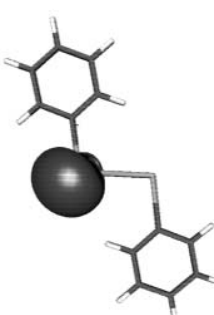

Lone Pair 2
Fig. 2 Localized Edminston-Ruedenberg orbitals for the planar singly bonded structure (structure I of Fig. 2).

If spin-symmetry is maintained (rather than broken as above) then the optimization yields a gauche like structure seen in Fig. 1 (structure II). This structure is very twisted and breaks the symmetry about the central $\mathrm{Sn}$ bond seen in the singly bonded structure. With a Sn-Sn bond length of $3.127 \AA$, the gauche structure would still be classified as a singly bonded structure, since a multiply bonded structure at this geometry would necessarily break the $\pi$-like bonds. This gauche structure has a dihedral angle $\omega=73.6^{\circ}$ and a Sn-Sn-C angles of 103.1 and 91.6. With a drastically different geometry, it is surprising that the gauche structure is only $0.3 \mathrm{kcal} \mathrm{mol}^{-1}$ less stable than the planar structure. This result implies that the potential energy surface for this compound is very flat, as was indeed already reported for the MeSnSnMe model.

A cross-section of the $\mathrm{PhSnSnPh}$ potential energy surface scanned with respect to dihedral angle is mapped out in Fig. 3. Several features of this surface are of particular interest. First, as already discussed is that the global minimum occurs at the planar geometry when spin symmetry is lifted. The next lowest point on the surface is the gauche structure which is only $0.3 \mathrm{kcal} \mathrm{mol}^{-1}$ higher in energy. Since there is only a very small

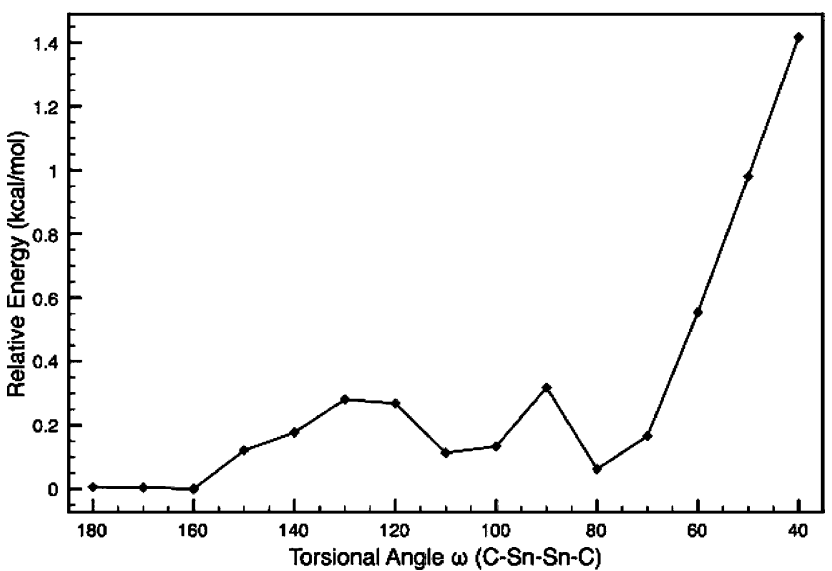

Fig. 3 Potential energy surface for $\mathrm{PhSnSnPh}$ with respect to dihedral angle changes.

Table 2 Structural details and energetics for each of the four structures shown in Fig. 1

\begin{tabular}{lllll}
\hline Structure & $\mathrm{Sn}-\mathrm{Sn} / \AA$ & $\theta{ }^{\circ}$ & $\omega^{\circ}$ & $\Delta E / \mathrm{kcal} \mathrm{mol}^{-1}$ \\
\hline I & 3.134 & 98.1 & 180 & - \\
II & 3.127 & $103.1 / 91.6$ & 73.6 & 0.3 \\
III & 2.77 & 122.3 & 179.9 & 7.6 \\
IV & 2.758 & 125.0 & 180 & 12.5 \\
\hline
\end{tabular}

energetic barrier, interconversion between these two structures should be facile. Finally, it should be noted that this surface is very shallow and bumpy, creating several local minima and a delicate global minimum, which could readily be perturbed by changes in substituents.

The model system can also adopt a geometry classified as a multiply bonded distannyne if we force the orbitals to be spinrestricted (in other words this is an unstable solution). The multiply bonded structure is not located on the surface pictured in Fig. 3 and is, in fact, $7.6 \mathrm{kcal} \mathrm{mol}^{-1}$ less stable than the planar singly bonded structure. This multiply bonded structure is planar with a Sn-Sn bond distance of $2.770 \AA$ and a $\theta=122.3^{\circ}$. These results along with the other structures are summarized in Table 2. The most interesting aspect of this structure is that the plane of the phenyl rings is perpendicular to the plane of the $\mathrm{Sn}-\mathrm{Sn}$ bond (Fig. 1 structure III). This result is somewhat surprising since this is not the same multiply bonded structure that is seen in the $\mathrm{Ar}^{\prime} \mathrm{SnSnAr}$ ' calculations or experimental structure. By looking at the Edmiston-Ruedenberg localized orbitals (Fig. 4), there are three orbitals that have bonding character, thereby confirming the $\mathrm{Sn}-\mathrm{Sn}$ multiple bond of this species (though only one is a true bonding orbital).

If the starting geometry of an optimization resembles a planar multiply bonded structure and the symmetry of the molecule is constrained to $C_{2 \mathrm{~h}}$, and if the orbitals are constrained to be spin-restricted, then (finally) it is possible to obtain an optimized planar structure that is multiply bonded with the phenyl rings in the plane of the $\mathrm{Sn}-\mathrm{Sn}$ bond instead of perpendicular. This structure is planar with a Sn-Sn bond distance of $2.758 \AA$ and $\theta=$ $125.0^{\circ}$ (Fig. 1 structure $\mathbf{I V}$ ). The Edmiston-Ruedenberg localized orbitals of this species are almost identical to those belonging to structure III (Fig. 5). This confirms that both structure III 

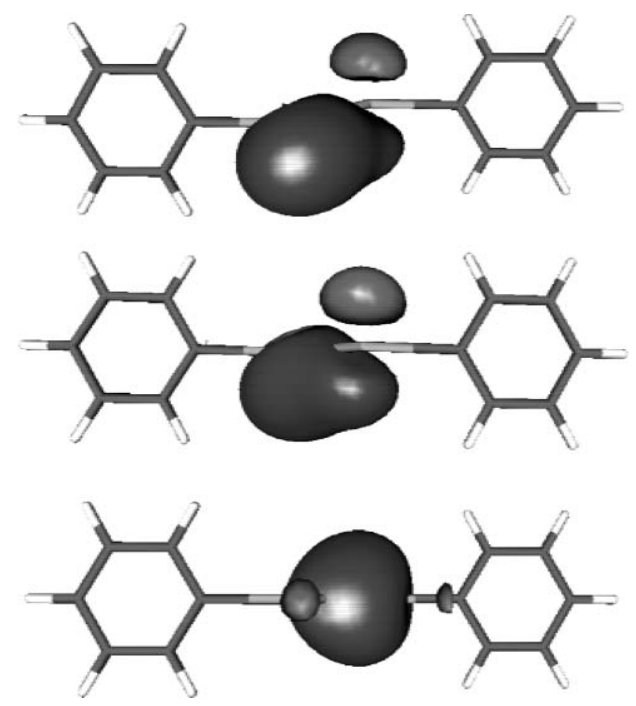

Fig. 4 The three Edmiston-Ruedenberg bonding orbitals between the two tin atoms in structure III of Fig. 1

and IV are similarly bonded and can be classified as multiply bonded. It should be noted that this structure is the highest energy $\mathrm{PhSnSnPh}$ structure being $12.5 \mathrm{kcal} \mathrm{mol}^{-1}$ less stable than the global minimum. This result is surprising since the full crystal $\mathrm{Ar}^{\prime} \mathrm{SnSnAr}$ ' structure adopts a ground state geometry very similar to this one. The notably large energetic differences between the full crystal structure and this model suggests that further inquiry into the stability and energetics of the full molecule is necessary (see final section).

It must be emphasized that these last two structures are only obtained if spin symmetry is enforced. If spin symmetry is lifted and these structures are re-optimized, they will collapse down to the planar global minimum (structure I). In other words, both structures are obtained with KS orbitals that are saddlepoints in the space of wavefunctions, rather than minima. Stability analysis on this structure confirms that these solutions display a triplet instabilities and a lower energy solution available when spin symmetry is lifted. Of course this does not mean that the multiply bonded solution is always a saddle-point - this can be influenced by further changes in substituents as we explore later.

Perfect pairing calculations were run on the four structures shown in Fig. 1 in order to determine the LUMO occupation numbers and therefore the amount of diradical character for each structure. Structures I and II both have similar diradical character with total LUMO occupations being about 0.05 for both structures. These numbers are in line with previous results that stated that a singly bonded structure should have a LUMO occupation of 0.04. Having a low LUMO occupation number is also consistent with the fact that these structures have the singly bonded characteristics of the diplumbynes. The multiply bonded structures III and IV have LUMO occupation numbers that are quite a bit larger at 0.16 and 0.12 , respectively, signifying between two and three times more diradical character. The higher diradical character for the multiply bonded structure (III) is also consistent with the earlier results for multiply bonded digermynes that showed a LUMO occupation number of about 0.13 electrons.

\section{Effect of electron-withdrawal and electron-donation}

Other structures were investigated for the purpose of further exploring electronic effects on distannynes. In order to investigate different charges, the model system was oxidized or reduced, and the structure fully reoptimized. In this series, the cation has the longest $\mathrm{Sn}-\mathrm{Sn}$ bond distance at $3.29 \AA$, followed by the neutral species with $3.13 \AA$, and the anion has the smallest $\mathrm{Sn}-\mathrm{Sn}$ bond distance at $3.02 \AA$. Both the anionic and neutral species have planar structures where the cation has a slightly puckered structure with the dihedral $\omega=160^{\circ}$. As the model system becomes more reduced, the system becomes more trans-bent with the angle of the cation being $\theta=103^{\circ}$ and the anion being $\theta=97^{\circ}$. All of these results are summarized in Table 3. By comparing the neutral species to the anion and the cation, it is fairly clear from a structural point of view that the neutral species resembles the anionic species more than the cationic species. It has been previously reported that reduced distannyne crystal salts take a singly bonded structure. Therefore, it should be no surprise that the planar singly bonded neutral species resembles the anionic species known to take a singly bonded geometry. The contraction in $\mathrm{Sn}-\mathrm{Sn}$ distance is also consistent with the behavior of triplet $\mathrm{MeSnSnMe}$ relative to singlet which as reported previously also showed a bond-length contraction. These results indicate that the LUMO of the neutral singlet species has partial bonding character. All of these species have fairly similar geometry, and would be classified as singly bonded species. The multiply bonded structure of this model system cannot be found as a global energy minimum by altering the oxidation state of the model system.

We performed additional calculations that further probe electronic effects using reasonably strong electron withdrawing (nitro) and electron donating (amino) groups as substituents instead of fully reducing or oxidizing the species. Both substituents create tin-nitrogen bonds, and therefore differences in these two structures cannot be ascribed to differences in their bonding

Table 3 Structural details concerning the anion, neutral, and cation of $\mathrm{PhSnSnPh}$

\begin{tabular}{llrl}
\hline Species & $\mathrm{Sn}-\mathrm{Sn} / \AA$ & $\theta /{ }^{\circ}$ & $\omega /^{\circ}$ \\
\hline$[\mathrm{PhSnSnPh}]^{-}$ & 3.022 & 96.7 & 180 \\
$\mathrm{PhSnSnPh}$ & 3.134 & 98.1 & 180 \\
[PhSnSnPh $^{+}$ & 3.293 & 103.2 & 160.1 \\
\hline
\end{tabular}
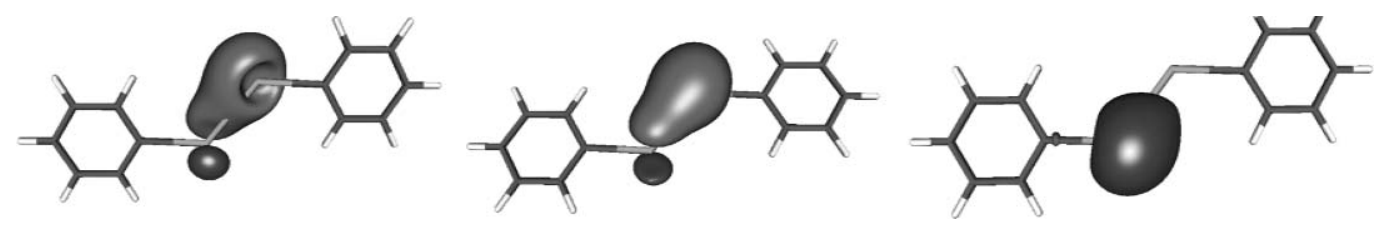

Fig. 5 The three Edmiston-Ruedenberg bonding orbitals between the two tin atoms in structure IV of Fig. 1. 
Table 4 Structural details concerning $\mathrm{RSnSnR}$ with $\mathrm{R}=\mathrm{Ph}, \mathrm{NH}_{3}, \mathrm{NO}_{2}$

\begin{tabular}{llcc}
\hline $\mathrm{R}$ & $\mathrm{Sn}-\mathrm{Sn} / \AA$ & $\theta /{ }^{\circ}$ & $\omega /{ }^{\circ}$ \\
\hline $\mathrm{Ph}$ & 3.134 & 98.1 & 180 \\
$\mathrm{NH}_{3}$ & 3.197 & 92.3 & 180 \\
$\mathrm{NO}_{2}$ & 3.12 & $104 / 71$ & -112.3 \\
\hline
\end{tabular}

to the central tin atoms. The amine structure is planar with a central angle of $\theta=92^{\circ}$, and an Sn-Sn bond distance of $3.20 \AA$, which would classify this species as a singly bonded structure, similar to the global minimum of $\mathrm{PhSnSnPh}$ discussed earlier. The nitro structure adopts a gauche geometry with the dihedral $\omega=-112^{\circ}$ and a Sn-Sn bond distance of $3.12 \AA$. Similar to the $\mathrm{PhSnSnPh}$ gauche structure, the nitro structure breaks symmetry with the central bond angles measuring $\theta=104^{\circ}$ and $71^{\circ}$. These results are summarized in Table 4 . Evidently electron withdrawing groups preferentially stabilize a gauche structure, whereas electron donating groups tend to stabilize a planar singly bonded structure. Similarly the contrasting global minima for MeSnSnMe (gauche) and $\mathrm{PhSnSnPh}$ (trans singly bonded) suggest that the phenyl rings are more electron-donating towards the SnSn moiety than methyl groups. We note in passing that the effects of phenyl substitution to the lead system $(\mathrm{PhPbPbPh})$ have been studied previously. ${ }^{13}$ There, both the steric and electronic effects of 2,6- $\mathrm{Ph}_{2} \mathrm{C}_{6} \mathrm{H}_{3}$ substituents were seen to play a role in stabilizing and making the singly bonded lead isomer a true energy minimum as in experiments.

\section{Comparison with experimentally synthesized molecules and discussion}

Preliminary calculations were done on the large $\mathrm{Ar}^{\prime} \mathrm{SnSnAr}^{\prime}\left(\mathrm{Ar}^{\prime}=\right.$ $\left.\mathrm{C}_{6} \mathrm{H}_{3}-2,6-\left(\mathrm{C}_{6} \mathrm{H}_{2}-2,6-\mathrm{Pr}_{2}^{\mathrm{i}}\right)_{2}\right)$ system in order to compare with experimental structural data and earlier calculations. These calculations using spin-restricted DFT (full details in ESI) confirmed the earlier findings ${ }^{15}$ that this structure does indeed take a multiply bonded structure, consistent with experiment. In particular, the optimized bond length is $2.832 \AA$ and the C-Sn-Sn bond angles are 128.2 and $130.7^{\circ}$. Even though the bond length is long enough to classify it as a single bond, the $\mathrm{C}-\mathrm{Sn}-\mathrm{Sn}$ angle is highly suggestive of a multiple bond. The key question raised by our model studies is whether or not this spin-restricted solution is stable or unstable to spin-polarization. Therefore we performed stability analysis at the optimized geometry, and, in striking contrast to the $\mathrm{PhSnSnPh}$ model, it was found that the spin-restricted orbitals are stable and no triplet instabilities occur.

Earlier computational results ${ }^{11}$ suggest that even bulky ligands can take the planar singly bonded structure as a global minimum. Both TbtSnSnTbt $\left(\mathrm{Tbt}=\mathrm{C}_{6} \mathrm{H}_{2}-2,4,6-\left[\mathrm{CH}\left(\mathrm{SiMe}_{3}\right)_{2}\right]_{3}\right)$ and Ar*SnSnAr* $\left(A r^{*}=\mathrm{C}_{6} \mathrm{H}_{3}-2,6-\left(\mathrm{C}_{6} \mathrm{H}_{2}-2,4,6-\mathrm{Pr}_{3}^{\mathrm{i}}\right)_{2}\right)$ were found to adopt a slightly puckered $\left(\omega \approx 170^{\circ}\right)$ singly bonded structure with Sn-Sn bond lengths over $3.00 \AA$ (albeit at a relatively low level of theory). Experimental evidence also suggests that the Ar*SnSnAr* and $\mathrm{Me}_{3} \mathrm{Si}-4-\mathrm{Ar}^{\prime} \mathrm{SnSnAr}-4-\mathrm{SiMe}_{3}$ structures both adopt a singly bonded structure. All of these structures contain a central core that have the central tin atoms bonded to aromatic carbon rings, so it is perhaps quite reasonable that $\mathrm{PhSnSnPh}$ also takes a singly bonded structure. On this basis, we suggest it is a reasonable model compound for understanding electronic effects in $\mathrm{Ar} * \mathrm{SnSnAr} *$ and $4-\mathrm{SiMe}_{3}-\mathrm{Ar}^{\prime} \mathrm{SnSnAr} \mathrm{Ar}^{\prime}-\mathrm{SiMe}_{3}$.

However, Ar'SnSnAr' does display the multiply bonded structure to be a local minimum within DFT, which is stable both with respect to spin-polarization, and with respect to nuclear displacements. This is in contrast to the $\mathrm{PhSnSnPh}$ model treated earlier at the same level of theory, where the $\mathbf{M}$ structure was unstable both with respect to nuclear displacements (the phenyl rings twisted perpendicular) and with respect to spinpolarization. The question of why the $\mathbf{M}$ structure arises with Ar' substituents is therefore of considerable interest. We had previously speculated that a preferred $\mathbf{S}$ structure was distorted to the $\mathbf{M}$ form due to crystal packing effects, but since isolated molecule optimizations yield an optimized M-type geometry that is in good agreement with the crystal structure, this cannot be the case. We must therefore conclude that there are additional intramolecular interactions associated with the $\mathrm{Ar}^{\prime}$ ligands used in the experimentally synthesized molecule that are not present in the $\mathrm{PhSnSnPh}$ model. It is an intriguing issue for the future to better characterize what these interactions are, and how they exert such a substantial differential effect on the relative stability of the singly and multiply bonded motifs. Separately, there remain some possible uncertainties and errors associated with the approximate DFT electronic structure methods used by all workers in this field, but they may not yet be limiting us in terms of understanding these systems.

\section{Conclusions}

In this report we have briefly reviewed a sometimes neglected aspect of density functional theory (DFT) electronic structure calculations that are relevant to characterizing potentially reactive heavy element congeners of the alkynes-specifically the role of spin-polarization and its detection by stability analysis. This proved to be the basis for a resolution of the striking discrepancy seen between two apparently similar sets of DFT calculations ${ }^{15,17}$ on the MeSnSnMe model of distannynes.

Seeking a better, yet still simple model for electronic interactions of experimental terphenyl substituents with the $\mathrm{SnSn}$ system, we then reported a detailed characterization of $\mathrm{PhSnSnPh}$. From this investigation we conclude that the singly bonded (S) structure is the global minimum of $\mathrm{PhSnSnPh}$. This global minimum can only be reached when spin-polarization is lifted. Other structures for distannynes exist as local minima on both the spin-polarized and the (sometimes unstable) spin-symmetric potential energy surface. These structures are all very close in energy so that any one of these structures can become the global minimum based on the electron donating and steric effects of the ligands attached directly to the tin atoms. Some of these possibilities were illustrated with different substitutions and oxidation states.

Finally, a comparison of the $\mathrm{PhSnSnPh}$ results with various experimentally isolated systems indicates that there are significant additional electronic effects associated with the terphenyl ligands. Some experimentally isolated species, such as $\mathrm{Ar} * \mathrm{SnSnAr} *$ and $\mathrm{Me}_{3} \mathrm{Si}-4-\mathrm{Ar}^{\prime} \mathrm{SnSnAr} \mathrm{H}^{\prime}-4-\mathrm{SiMe}_{3}$, exhibit $\mathbf{S}$-type structures that are reasonably consistent with $\mathrm{PhSnSnPh}$ (and indeed with $\mathrm{MeSnSnMe}$ as well). However, we find that, in contrast to $\mathrm{PhSnSnPh}$, no spin-polarization occurs for the multiply-bonded (M) structure of $\mathrm{Ar}^{\prime} \mathrm{SnSnAr}^{\prime}\left(\mathrm{Ar}^{\prime}=\mathrm{C}_{6} \mathrm{H}_{3}-2,6\left(\mathrm{C}_{6} \mathrm{H}_{2}-2,6-\mathrm{Pr}_{2}{ }_{2}\right)_{2}\right)$, 
which becomes a stable local minimum, consistent with the experimental geometry. Since loss of spin-polarization should be considered a consequence of electronic stabilization, and as through-bond interactions associated with $\mathrm{Ar}^{\prime}$ should not be substantially different to $\mathrm{Ph}$ itself, it appears that, intriguingly, we still have more to learn about the specific intramolecular interactions in this species.

\section{Acknowledgements}

This work was supported by the Director, Office of Energy Research, Office of Basic Energy Sciences, Chemical Sciences Division of the U. S. Department of Energy under Contract DEAC0376SF00098, with additional support through the Scientific Discovery through Advanced Computing (SciDAC) program of the Department of Energy. We acknowledge a grant of supercomputing time from NERSC.

\section{References}

1 P. P. Power, Appl. Organomet. Chem., 2005, 19, 488.

2 A. Sekiguchi, M. Ichinohe and R. Kinjo, Bull. Chem. Soc. Jpn., 2006, 79, 825 .

3 P. P. Power, Organometallics, 2007, 26, 4362.

4 L. H. Pu, B. Twamley and P. P. Power, J. Am. Chem. Soc., 2000, 122, 3524.

5 A. D. Phillips, R. J. Wright, M. M. Olmstead and P. P. Power, J. Am. Chem. Soc., 2002, 124, 5930.

6 M. Stender, A. D. Phillips, R. J. Wright and P. P. Power, Angew. Chem., Int. Ed., 2002, 41, 1785.

7 A. Sekiguchi, R. Kinjo and M. Ichinohe, Science, 2004, 305, 1755.

8 R. C. Fischer, L. H. Pu, J. C. Fettinger, M. A. Brynda and P. P. Power, J. Am. Chem. Soc., 2006, 128, 11366.

9 G. H. Spikes, J. R. Giuliani, M. P. Augustine, I. Nowik, R. H. Herber and P. P. Power, Inorg. Chem., 2006, 45, 9132.

10 C. M. Cui, M. M. Olmstead, J. C. Fettinger, G. H. Spikes and P. P. Power, J. Am. Chem. Soc., 2005, 127, 17530.

11 N. Takagi and S. Nagase, Organometallics, 2001, 20, 5498.

12 K. Kobayashi, N. Takagi and S. Nagase, Organometallics, 2001, 20, 234.

13 Y. Chen, M. Hartmann, M. Diedenhofen and G. Frenking, Angew. Chem., Int. Ed., 2001, 40, 2052.

14 M. Lein, A. Krapp and G. Frenking, J. Am. Chem. Soc., 2005, 127, 6290.

15 N. Takagi and S. Nagase, Organometallics, 2007, 26, 469.

16 N. Takagi and S. Nagase, Organometallics, 2007, 26, 3627.
17 Y. Jung, M. Brynda, P. P. Power and M. Head-Gordon, J. Am. Chem. Soc., 2006, 128, 7185.

18 R. G. Parr and W. Yang, Density-Functional Theory of Atoms and Molecules, Oxford University Press, Oxford-New York, 1989.

19 W. Kohn, A. D. Becke and R. G. Parr, J. Phys. Chem., 1996, 100, 12974.

20 J. A. Pople, P. M. W. Gill and N. C. Handy, Int. J. Quantum Chem., 1995, 56, 303.

21 J. Cizek and J. Paldus, J. Chem. Phys., 1967, 47, 3976.

22 R. Seeger and J. A. Pople, J. Chem. Phys., 1977, 66, 3045.

23 R. Bauernschmitt and R. Ahlrichs, J. Chem. Phys., 1996, 104, 9047.

24 Y. S. Jung, Y. H. Shao, M. S. Gordon, D. J. Doren and M. HeadGordon, J. Chem. Phys., 2003, 119, 10917.

25 Y. Shao, L. F. Molnar, Y. Jung, J. Kussmann, C. Ochsenfeld, S. T. Brown, A. T. B. Gilbert, L. V. Slipchenko, S. V. Levchenko, D. P. O’Neill, R. A. DiStasio, R. C. Lochan, T. Wang, G. J. O. Beran, N. A. Besley, J. M. Herbert, C. Y. Lin, T. Van Voorhis, S. H. Chien, A. Sodt, R. P. Steele, V. A. Rassolov, P. E. Maslen, P. P. Korambath, R. D. Adamson, B. Austin, J. Baker, E. F. C. Byrd, H. Dachsel, R. J. Doerksen, A. Dreuw, B. D. Dunietz, A. D. Dutoi, T. R. Furlani, S. R. Gwaltney, A. Heyden, S. Hirata, C. P. Hsu, G. Kedziora, R. Z. Khalliulin, P. Klunzinger, A. M. Lee, M. S. Lee, W. Liang, I. Lotan, N. Nair, B. Peters, E. I. Proynov, P. A. Pieniazek, Y. M. Rhee, J. Ritchie, E. Rosta, C. D. Sherrill, A. C. Simmonett, J. E. Subotnik, H. L. Woodcock, W. Zhang, A. T. Bell, A. K. Chakraborty, D. M. Chipman, F. J. Keil, A. Warshel, W. J. Hehre, H. F. Schaefer, J. Kong, A. I. Krylov, P. M. W. Gill and M. Head-Gordon, Phys. Chem. Chem. Phys., 2006, 8, 3172.

26 A. D. Becke, J. Chem. Phys., 1993, 98, 5648.

27 R. H. Hertwig and W. Koch, Chem. Phys. Lett., 1997, 268, 345.

28 P. M. W. Gill, B. G. Johnson and J. A. Pople, Chem. Phys. Lett., 1993, 209, 506.

29 C. S. Nash, B. E. Bursten and W. C. Ermler, J. Chem. Phys., 1997, 106, 5133.

30 S. Huzinaga, J. Andzelm, M. Klobukowski, E. Radzio-Andzelm, Y. Sakai and H. Tatewaki, Gaussian Basis Sets for Molecular Calculations, Elsevier, Amsterdam, 1984.

31 J. Cullen, Chem. Phys., 1996, 202, 217.

32 A. Sodt, G. J. O. Beran, Y. S. Jung, B. Austin and M. Head-Gordon, J. Chem. Theory Comput., 2006, 2, 300.

33 L. Salem and C. Rowland, Angew. Chem., Int. Ed. Engl., 1972, 11, 92.

34 D. Dohnert and J. Koutecky, J. Am. Chem. Soc., 1980, 102, 1789.

35 M. S. Gordon, M. W. Schmidt, G. M. Chaban, K. R. Glaesemann, W. J. Stevens and C. Gonzalez, J. Chem. Phys., 1999, 110, 4199.

36 Y. S. Jung and M. Head-Gordon, J. Phys. Chem. A, 2003, 107, 7475.

37 C. Edmiston and K. Ruedenberg, Rev. Mod. Phys., 1963, 35, 457.

38 J. E. Subotnik, Y. H. Shao, W. Z. Liang and M. Head-Gordon, J. Chem. Phys., 2004, 121, 9220.

39 J. E. Subotnik, A. Sodt and M. Head-Gordon, Phys. Chem. Chem. Phys., 2007, 9, 5522.

40 F. Weigend and R. Ahlrichs, Phys. Chem. Chem. Phys., 2005, 7, 3297.

41 F. Weigend, M. Haser, H. Patzelt and R. Ahlrichs, Chem. Phys. Lett., 1998, 294, 143. 